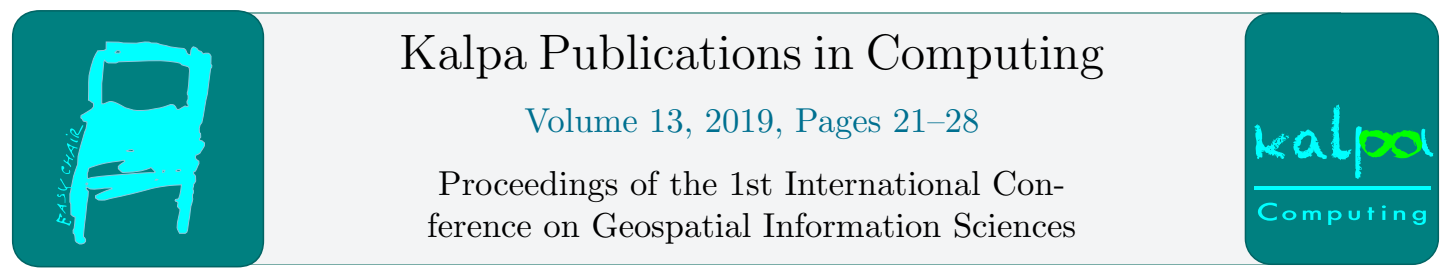

\title{
Potential distribution of clandestine graves in Guerrero using geospatial analysis and modelling
}

\author{
José L Silván-Cárdenas ${ }^{1 *}$, Ana Alegre-Mondragón² ${ }^{2}$, and Karime \\ González-Zuccolotto ${ }^{3}$ \\ 1 CentroGeo CDMX, CDMX, México. \\ jsilvan@centrogeo.edu.mx \\ 2 jalegre@centrogeo.edu.mx \\ 3 karime.gonzalez@centrogeo.edu.mx
}

\begin{abstract}
Searching clandestine graves is a huge task being conducted by many people around the world. In Mexico, this activity has steadily grown since the disappearance of the 43 students from Ayotzinapa, Gro. leading to the discovery of over a hundred of clandestine graves in the vicinity of Iguala, Gro. In order to facilitate extensive searches, a map of the potential distribution of clandestine graves would be valuable as it can reduce time, cost and effort paid by search brigades. This paper introduces the concept of clandestine space, shows its relation with known grave locations and uses it to map the potential distribution of clandestine graves in Guerrero by means of a machine learning approach.
\end{abstract}

\section{Introduction}

In the recent years, an alarming number of human remains have been encountered within clandestine graves around the country. Only since the authorities began the search for the 43 Ayotzinapa normalists who disappeared on September 26 of 2014, over a hundred graves were located in the vicinity of Iguala, Guerrero. However, the locations of these clandestine graves were mainly determined from witness statements, whereas in the vast majority of cases the lack of witnesses or their fear of testifying in general makes it difficult to locate or even know about their existence. It is believed that an even larger number of graves may be awaiting for discovery given the large number of disappeared people across the country. The most recent official figure of missing people in Mexico was released last January by the National People Search Commission, according to which there are more than 40 thousand 180 Mexicans disappeared since 2006 .

It has been under such scenario that many organized search groups around the country -formed mainly by family members of disappeared people- have developed a rudimentary field exploration technique, known as "envarillado", that consists of introducing a rod (of around $1.2-\mathrm{cm}$ thick by $1.5-2.20-\mathrm{m}$ length) on the ground floor. If there are bodies under the surface,

${ }^{*}$ Corresponding author

O. S. Siordia, J.L. Silván Cárdenas, A. Molina-Villegas, G. Hernandez, P. Lopez-Ramirez, R. Tapia-McClung, K. González Zuccolotto and M. Chirinos Colunga (eds.), iGISc 2019 (Kalpa Publications in Computing, vol. 13), pp. 21-28 
the tip pierces tissue or bumps into bones, and then the smell of death emanating from the ground is the irrefutable sign of the discovery of a clandestine grave. At the international level, there are also some technologies that can help the search for human remains below the surface in a range between 0 and 10 meters deep. Among these technologies are resistivity profilers, magnetometry profilers, thermography cameras and ground penetration radars [3, 7, 10, 11].

All above referred methods require a fairly intense field deployment and can only cover small areas, so they require significant delimitation of the search area. More recently, remote sensing techniques, such as LiDAR and hyperspectral cameras on board of either terrestrial platforms, aircrafts or unmanned aerial vehicles (UAV), have been suggested for cases under very specific circumstances $[6,13,2,4]$, yet the covered area is still limited to a few kilometers due to the high cost of data acquisition and to the high computing power required for data analysis.

In light of the above, methods to narrow down the search space are needed. In this study, we used satellite imagery and relevant geographical layers to model the potential distribution of grave locations in the vicinity of Iguala, Guerrero. In the rest of the paper we first introduce the concept of clandestine space and propose a way to measure it using geospatial modelling, then we investigate the relation of the clandestine space with known grave locations and use it to develop a model of the potential distribution of clandestine graves along a fairly extended area.

\section{Space conceptualization}

The illegal nature of clandestine graves forces creators to choose hidden sites, such as canyons or wooded areas, to guarantee their privacy during burial creation. Given such privacy requirement, the landscape structure, conformed mainly by terrain and vegetation, must play a crucial role in the election of a burial site. On the other hand, the geographical context imposes accessibility restrictions, specially because the movement of people against their will, or of human remains, requires fast and discrete transportation, so that transportation network and terrain slope must play an important role. These notions led us to conceptualize the geographical space in terms of two key concepts, namely the spatial accessibility and the spatial privacy that must be relevant to clandestine grave locations.

In the next section we show how to quantify these two concepts, but for now let us accept they are quantifiable in, at least an ordinal scale, so they define dimensions of analysis that allow us to partition the geographical space into four classes as outlined in Fig. 1. Areas of large accessibility and low privacy are referred to as the public space whereas areas with low accessibility and large privacy define the private space. In between these two extreme cases there are two other combinations, namelly, the scenic space having jointly low accessibility and low privacy, and the clandestine space having jointly high accessibility and high privacy. We hypothesize that clandestine graves are more likely to be found in the latter, and therefore a delineation of such kind of spaces will provide a prioritization scheme for search tasks.

\section{Geospatial modelling}

\subsection{Accessibility}

Spatial accessibility refers to how easy a site can be reached. Depending on the scale of analysis and available information of the space occupancy, one may measure accessibility in several different ways. For the regional study in hand, one intuitive measure is the travel time from 


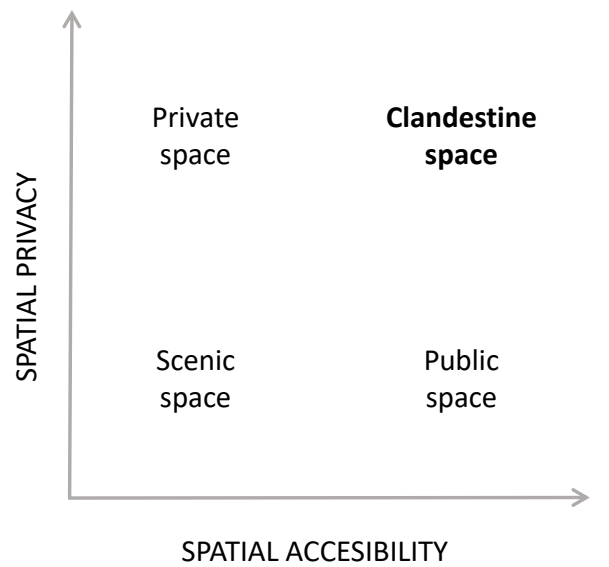

Figure 1: Conceptual model of the geographical space for clandestine graves search (see the text).

the nearest urban settlement. Urban settlements are used as departure points because they are where the largest amount of crime activity originates. The travel time is indeed a reverse measure of accessibility so that the lower the time, the higher the accessibility and vice versa.

Travel time can be computed for all sites (cells or pixels) of a geographical area through a cumulative cost mapping method from graph theory [1], where each pixel of the cost map gives the total cost of the lowest cost path to the nearest source location, that is, the accumulated time of the shortest path from nearest urban location. The cost function at the cell $i$ is given by the time per meter to traverse it, which can be modelled as follows:

$$
f_{i}=\frac{1}{v_{i} \cos \phi_{i}}
$$

where $\phi_{i}$ and $v_{i}$ are the terrain slope and the speed at the cell. It should be noted that the denominator of Eq. (1) expresses the horizontal speed, so that a 90-degree slope represents a barrier because it takes an infinity time to transverse such a cell.

Cells corresponding to roads will have speeds assigned in terms of allowed maximum speed of the road segment $\left(v_{\text {road }}\right)$. However, for all other non-road cells, a movement model needs to be implemented. In absence of road, people must use a transportation mean other than vehicles; they typically walk. The simplest model would be to use the typical walking speed of $5 \mathrm{~km} / \mathrm{h}$. Nonetheless, vegetation may also be used as a constraint. Here we implemented the following linear model of walking speed:

$$
v_{w a l k}=5(1-\alpha)
$$

where $\alpha$ is the fraction of vegetation cover of the cell.

\subsection{Privacy}

The spatial privacy is inversely related to visibility of a site, where visibility is here defined as the probability of being visible from any accessible point. At the region scale, accessible 
points are the road locations, so that visibility is mainly determined by the form of the terrain and vegetation abundance, whereas artificially induced privacy from human infrastructure and buildings is disregarded.

Viewshed analysis provides a methodological way to approach spatial privacy. A viewshed is the area that is visible from a given point over a digital elevation model [5]. Unfortunately, computing a viewshed is computational intensive as it requires to determine a line of sight from each pixel to the observer location. This represents a limitation because estimating the probability of being visible from roads demands computing a large number of viewsheds. For instance, if we were to use all the vertices of the road network as the oberver's location in our study area (around 1.2 million vertices) it would take a standard PC of the time more than two years to compute such an amount of viewsheds! Therefore, the visibility map can be only approximated by limiting the observation distance and the number of observer's locations.

Another factor contributing to visibility is vegetation cover. Although site visibility from a given point depends on 3-d vegetation structure and its distribution along the line of sight, at the moderate resolution of $30 \mathrm{~m}$ per cell, it suffices to use the cover fraction of the observed cell. Hence, given $N$ sample points of the road network, the visibility map is computed by accumulating the viewshed masks over all observer's locations $x_{j}$ within the observation range $r$, normalized by the total number of observations, times the gap fraction of vegetation, or otherwise stated:

$$
V i s_{i}=\left(1-\alpha_{i}\right) \frac{\sum_{j}^{N} V S_{i}\left(x_{j}, r\right)}{\sum_{j}^{N} C_{i}\left(x_{j}, r\right)}
$$

where $V S_{i}\left(x_{j}, r\right)$ denotes the viewshed from point $x_{j}, C\left(x_{j}, r\right)$ is the circle mask centered at $x_{j}$ and $\alpha_{i}$ is, as before, the fraction of vegetation cover at the site.

\subsection{Distribution}

The potential distribution of clandestine graves was modeled using the maximum entropy model $[8,9]$. This is a machine learning method that is typically used for mapping species distribution based on environmental factors specified in a series of variables and a set of presence points for each species. The model expresses the suitability of a site for the existence of the species based on the environmental characteristics of the site. As such, it comprises a probabilistic model of maximum entropy subject to the average of the distribution being equal to the average of the data, where the underlying hypothesis is that environmental factors influence the distribution of species. There is nothing in this model, however, that limits its use to mapping species only.

Therefore, the MaxENT program coded by Steven Phillips, Miro Dudik and Rob Schapire $[8,9]$ was used with the dimensions of the so-called clandestine space, that is the travel time and visibility, as the major explanatory variables.

\section{Study area and data used}

The study area comprises a central segment of the mexican state of Guerrero that includes the cities of Iguala, Chilpancingo and Acapulco. In this area, 423 graves had been processed by the FGR since the kidnapping of the 43 students of Ayotzinapa in September of 2014, of which 142 were labelled as POSITIVE for a clandestine grave, meaning that human remains were found at those locations. The remaining 281 points were labelled as NEGATIVE for a clandestine grave (Non-grave). Figure 2 shows the distribution of such locations within the study area. 


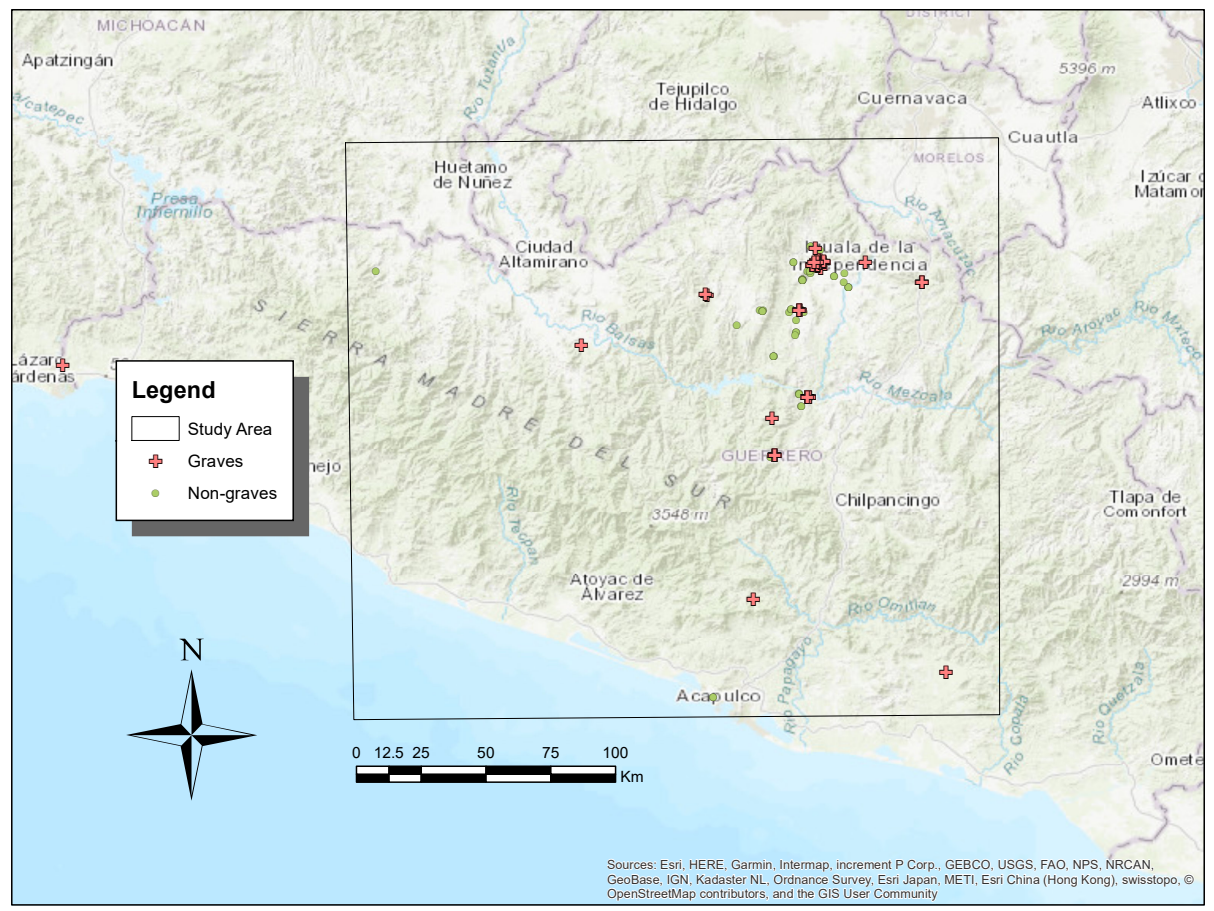

Figure 2: Postive and negative clandestine grave locations processed by the FGR within the study area.

Data used in this study is summarized in Table 1. The travel time map was computed in Google Earth Engine ${ }^{\mathrm{TM}}$ using the cumulative cost analysis tool with a mask of urban settlements generated by thresholding nighttime light imagery (DMSP-OLS) as the origin points, the road networks of the IMT with specified speed limit, the tree cover fraction generated by GLCF from Landsat imagery [12] and the slope computed from the SRTM digital elevation model.

The visibility map was generated in MATLAB ${ }^{\mathrm{TM}}$ using the viewshed function of the Mapping Toolbox. A script was coded to compute the visibility according to Eq. (2), where the SRTM digital elevation model, the GLCF tree cover fraction and up to 100,000 random points drawn from the road network were used. The visibility range of every point was limited to $5 \mathrm{~km}$, which reduced significantly the computation time for the whole area to a little more than 24 hours.

\section{$5 \quad$ Results}

Figure 3 shows the scatterplot of travel time against visibility of known clandestine grave and non-grave locations. Considering the conceptual model of Figure 1, but with the scenic space and the clandestine space interchanged -remember that the accessibility and privacy had been measured in reverse way through the travel time and visibility, respectively- one can conclude that there are high chances to located graves in the clandestine space. More specifically, $94 \%$ of grave locations had a travel time under one hour, whereas $92 \%$ of them had a visibility under $50 \%$. On the other hand, one should not expect negative points to be exclusively outside 
Table 1: Datasets used in this study.

\begin{tabular}{|c|c|c|c|c|}
\hline Data & Source & Year & Res. & Derived layer \\
\hline Nightime images & NOAA/DMSP-OLS & 2013 & $1 \mathrm{~km}$ & urban mask \\
\hline Digital elevation model & USGS/SRTM & 2000 & $30 \mathrm{~m}$ & slope, terrain surface \\
\hline Tree cover percent & GLCF/Landsat & 2010 & $30 \mathrm{~m}$ & gap fraction \\
\hline National road network & IMT & 2018 & NA & travel time, observer's location \\
\hline Grave locations & FGR & 2016 & NA & model fitting/testing \\
\hline \multicolumn{5}{|c|}{ IMT - Instituto Mexicano del Transporte } \\
\hline \multicolumn{5}{|c|}{ FGR - Fiscalía General de la República } \\
\hline \multicolumn{5}{|c|}{ USGS - United States Geological Survey } \\
\hline \multicolumn{5}{|c|}{ GLCF - Global Land Cover Facility } \\
\hline \multicolumn{5}{|c|}{ NOAA - National Oceanic and Atmospheric Administration } \\
\hline \multicolumn{5}{|c|}{ SRTM - Shuttle Radar Topographic Mission } \\
\hline \multicolumn{5}{|c|}{ DMSP-OLS - Defense Meteorological Satellite Program-Operational Line Scan } \\
\hline
\end{tabular}

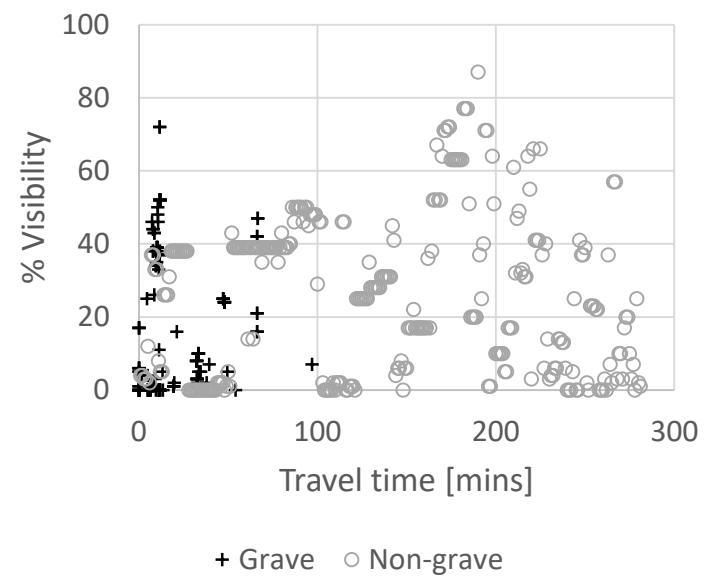

Figure 3: Travel time vs. visibility of actual clandestine graves and non-grave sites.

the clandestine space because there is not a grave at every location of the clandestine space . Nonetheless, in this case $6 \%$ of non-graves had a travel time beyond one hour and $14 \%$ had a visibility larger than $50 \%$. This is already a good result considering that the data had not been used to calibrate any model so far.

The grave points were used to train a MaxENT model, which was then used to map the potential distribution of clandestine graves over the entire study area. A close look to the map around the Iguala city area is shown in Figure 4. The polygon of an area that had been delineated independently by an expert analyst is also included for comparison purpose. Such an area was delineated taking into account sites of interest, such as unpaved roads, known clandestine grave locations, location of communication antennas with a report of more frequent use during the kidnapping of the students, locations of homes and places frequented by people involved in the criminal acts, as well as the natural limits imposed by the highlands and mountains in the northern fringe, and the limits of the urban areas on the south. Interestingly, most of the high probability area (pink and red tones) are included in the expert's, yet the latter also includes a large portion of moderate and low probability areas. Furthermore, the model included sev- 


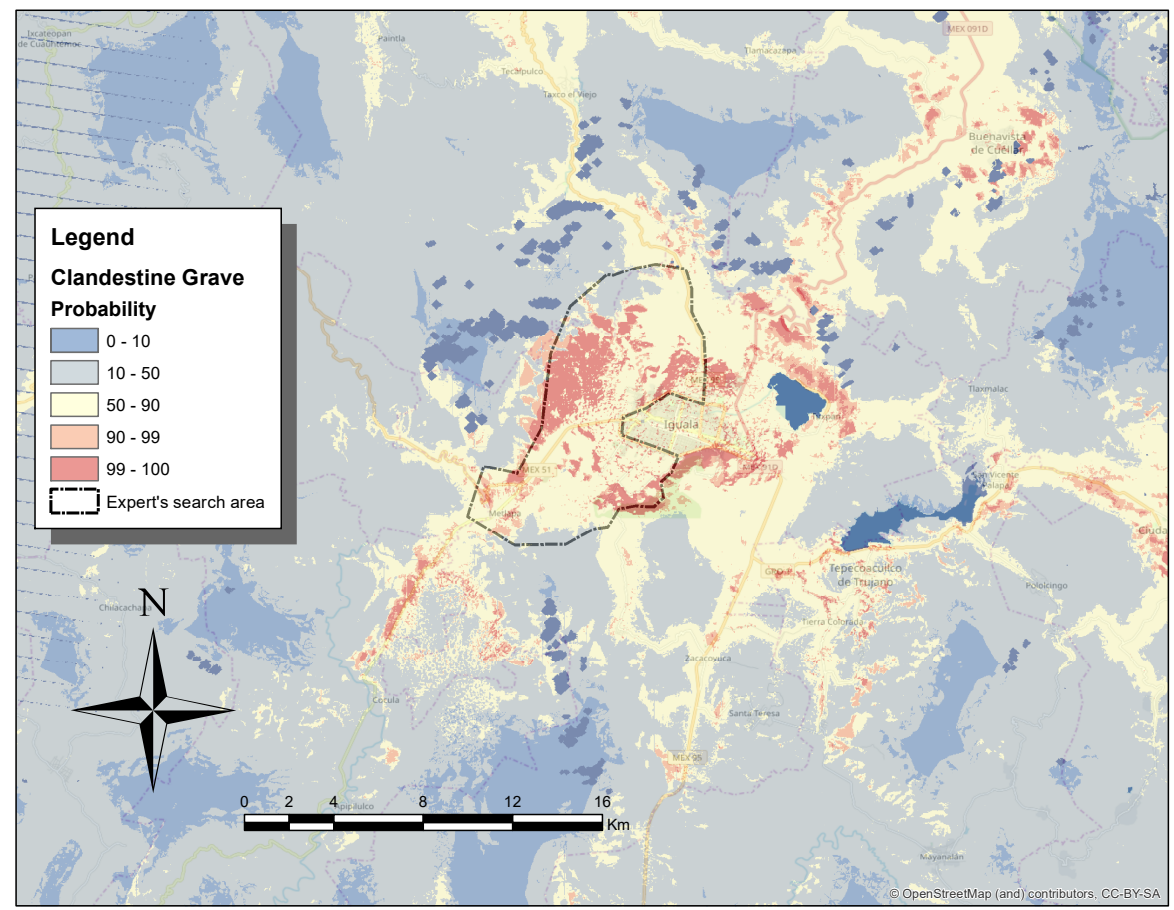

Figure 4: Potential distribution of clandestine graves around Iguala, Guerrero.

eral important areas further east and south of the city that were not included in the expert's polygon.

\subsection{Conclusions}

The concept of clandestine space was defined as the space with a jointly high spatial accessibility and high spatial privacy. These two dimensions were, in turn, inversely measured through the travel time from the nearest urban settlement and through the probability of being visible from roads, respectively. When analysing these measures for actual grave sites, we found that these had consistently low values suggesting that the so-defined clandestine space has high chances to include grave locations.

Then, we used these measures as the input of an statistical model to map the probability of graves distribution around the city of Iguala, Guerrero, and propose the high-probability areas (e.g., greater than 90\%) as the target area for the search tasks. Interestingly, an area generated independently by an expert analyst included must of such high probability areas, but the model also discarded a large amount of selected area and included some areas not considered by the expert.

Overall, this paper have shown how the conceptualization of the geographical space in terms of the fundamental requirements for creating a clandestine grave can lead to a better prioritization of search areas through geospatial modeling. While field exploration techniques can now be deployed on the highest probability areas, further research involving higher resolution data along the high-probability areas and other sources of information specific to the Ayotzinapa 
case may also be conducted in order to further refine the wider areas.

\section{Acknowledgments}

Graves locations used in this study were provided by the Comisión para la Verdad y acceso a la Justica para el Caso Ayotzinapa. This work is dedicated to all the disappeared people in Mexico.

\section{References}

[1] Frank Adriaensen, JP Chardon, Geert De Blust, Else Swinnen, S Villalba, Hubert Gulinck, and Erik Matthysen. The application of 'least-cost'modelling as a functional landscape model. Landscape and urban planning, 64(4):233-247, 2003.

[2] Katie A Corcoran, Amy Z Mundorff, Devin A White, and Whitney L Emch. A novel application of terrestrial lidar to characterize elevation change at human grave surfaces in support of narrowing down possible unmarked grave locations. Forensic science international, 289:320-328, 2018.

[3] G Clark Davenport. Remote sensing applications in forensic investigations. Historical Archaeology, 35(1):87-100, 2001.

[4] León Dozal, José L Silván-Cárdenas, Daniela Moctezuma, Oscar S Siordia, and Enrique Naredo. Evolutionary approach for detection of buried remains using hyperspectral images. Photogrammetric Engineering \& Remote Sensing, 84(7):435-450, 2018.

[5] Peter F Fisher. Algorithm and implementation uncertainty in viewshed analysis. International Journal of Geographical Information Science, 7(4):331-347, 1993.

[6] Margaret E Kalacska, Lynne S Bell, G Arturo Sanchez-Azofeifa, and Terry Caelli. The application of remote sensing for detecting mass graves: an experimental animal case study from costa rica. Journal of forensic sciences, 54(1):159-166, 2009.

[7] Peter S Miller. Disturbances in the soil: finding buried bodies and other evidence using ground penetrating radar. Journal of Forensic Science, 41(4):648-652, 1996.

[8] Steven J Phillips, Robert P Anderson, and Robert E Schapire. Maximum entropy modeling of species geographic distributions. Ecological modelling, 190(3-4):231-259, 2006.

[9] Steven J Phillips and Miroslav Dudík. Modeling of species distributions with maxent: new extensions and a comprehensive evaluation. Ecography, 31(2):161-175, 2008.

[10] Kathryn Powell. Detecting buried human remains using near-surface geophysical instruments. Exploration Geophysics, 35(1):88-92, 2004.

[11] JK Pringle, A Ruffell, JR Jervis, L Donnelly, J McKinley, J Hansen, R Morgan, D Pirrie, and M Harrison. The use of geoscience methods for terrestrial forensic searches. Earth-Science Reviews, 114(1-2):108-123, 2012.

[12] Joseph O Sexton, Xiao-Peng Song, Min Feng, Praveen Noojipady, Anupam Anand, Chengquan Huang, Do-Hyung Kim, Kathrine M Collins, Saurabh Channan, Charlene DiMiceli, et al. Global, 30-m resolution continuous fields of tree cover: Landsat-based rescaling of modis vegetation continuous fields with lidar-based estimates of error. International Journal of Digital Earth, 6(5):427-448, 2013.

[13] José Luis Silván-Cárdenas, Nirani Corona-Romero, José Manuel Madrigal-Gómez, Aristides Saavedra-Guerrero, Tania Cortés-Villafranco, and Erick Coronado-Juárez. On the detectability of buried remains with hyperspectral measurements. In Mexican Conference on Pattern Recognition, pages 201-212. Springer, 2017. 